\title{
Signal Regeneration Techniques for Advanced Modulation Formats
}

\author{
Radan Slavík $^{1 *}$, Joseph Kakande ${ }^{2}$, Francesca Parmigiani ${ }^{1}$, Periklis Petropoulos ${ }^{1}$, and David J. Richardson ${ }^{1}$ \\ 1. Optoelectronics Research Centre, University of Southampton, Southampton, SO17 1BJ, UK \\ 2. Bell Labs, Alcatel-Lucent, Holmdel, NJ 07733, USA \\ *r.slavik@soton.ac.uk
}

\begin{abstract}
We review recent results on the regeneration of phase encoded (BPSK, QPSK) signals and discuss paths towards regeneration of amplitude/phase encoded (QAM) signals. Regeneration based on phase sensitive amplification will be discussed in detail.

(C)2013 Optical Society of America

OCIS codes: (060.2320) Fiber optics amplifiers and oscillators, (190.4410) Nonlinear optics, parametric processes
\end{abstract}

\section{Introduction}

Phase noise introduced during transmission both from optical amplifiers and the nonlinear interactions between channels represents a significant limiting factor to data transmission using (differential) phase-shift keyed ((D)PSK), modulation formats [1]. Consequently, there is great interest in developing all-optical regeneration schemes capable of eliminating phase and amplitude noise for phase shift keyed signals.

Phase regenerators can be designed to operate either directly on the optical field, or by first converting the optical field into an intensity based representation - e.g., into two intensity-only modulated data streams, one carrying information about the optical phase and the other about the intensity. Subsequently, this second class of regenerator acts on the converted signals (via more mature intensity regeneration approaches) and eventually remodulates the information back onto a single (complex) data stream. There is also a third category of optical regenerators which even though they regenerate the amplitude only and not the phase, they can still reduce amplitude-to-phase noise conversion in the subsequent propagation (so called phase-preserving amplitude regeneration).

Regenerators operating directly on the optical field are generally based on Phase Sensitive Amplification (PSA) [2] and have been demonstrated so far only for phase-only modulated signals (binary PSK, BPSK; quadruple PSK, QPSK) [3-5]. We will discuss their principles, putting particular emphasis on their broader features and capabilities and review the key results obtained so far. We will also discuss routes towards multiple-channel operation (e.g., [6]) and operation over multiple amplitude levels (discussing routes towards QAM modulation).

\section{Optical Vector Processing Toolbox}

Traditional, simple PSA can only be used for regeneration of BPSK signals. In our work, in which we concentrated on PSA using Four Wave Mixing (FWM) in optical fibers, we found that by simultaneously employing multiple FWM processes, more complex modulation formats (e.g. QPSK) can be regenerated. To get a deeper and broader understanding, and to appreciate also other possible applications, we present this technology in terms of an Optical Vector Processing Toolbox, Fig. 1. As we see, FWM allows us to generate phase harmonics of our signal $\left( \pm 1^{\text {st }}, \pm 2^{\text {nd }}\right.$, ...) through Field Conjugation and Phase Multiplication and to sum them (via Field Addition) and eventually set their amplitudes (via Magnitude scaling). This represents Fourier synthesis which in principle allows any transfer characteristics to be obtained - we generate the signal harmonics first and then perform the summation of these harmonics. It is worth mentioning that the Coherent Addition is generally referred to as Phase Sensitive Amplification, as the output intensity of the signal and idler is dependent on their relative phases [2].

For the sake of simplicity, we show an example of how to regenerate QPSK signals, Fig. 2. Four-level phase regeneration $(M=4)$ requires a stair-case phase transfer function having four steps [5]. In [4] it was proposed to achieve this by interfering the signal with a conjugated $3^{\text {rd }}$ phase harmonics. After generating the $3^{\text {rd }}$ harmonics, the original signal ( $1^{\text {st }}$ harmonics) and the $3^{\text {rd }}$ harmonics were coherently added in a 2-pump non-degenerate PSA, Fig. 2. By analysing the phase condition of this PSA it is found that a signal with a nominal phase of $0,90,180,270 \mathrm{deg}$ (which is where the data are) is amplified, while a signal with a phase of 45, 135, 225, $315 \mathrm{deg}$ (where there is only noise) is de-amplified. This is exactly what is needed for QPSK regeneration. 


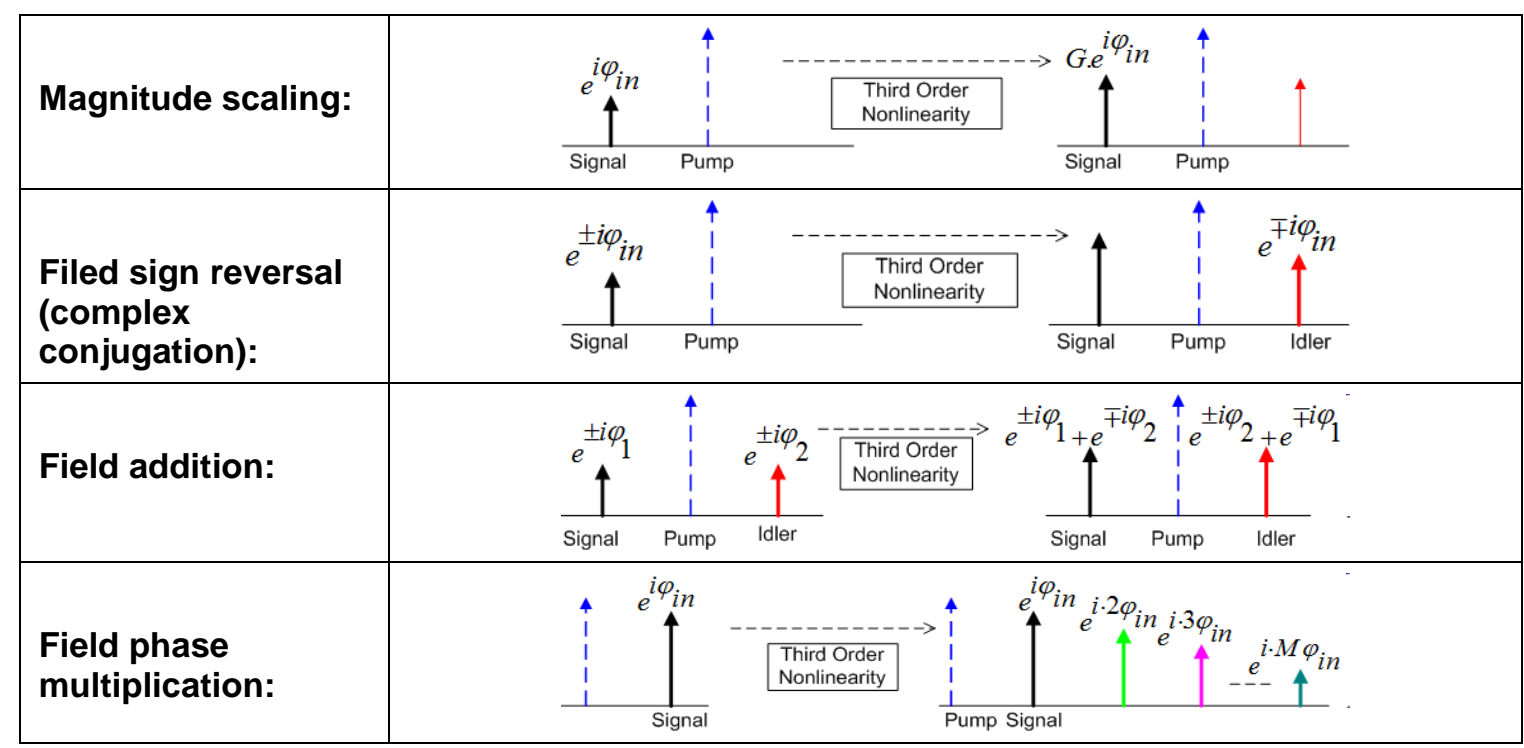

Fig. 1. Optical vector processing allows for synthesis of all Fourier components and their summation.

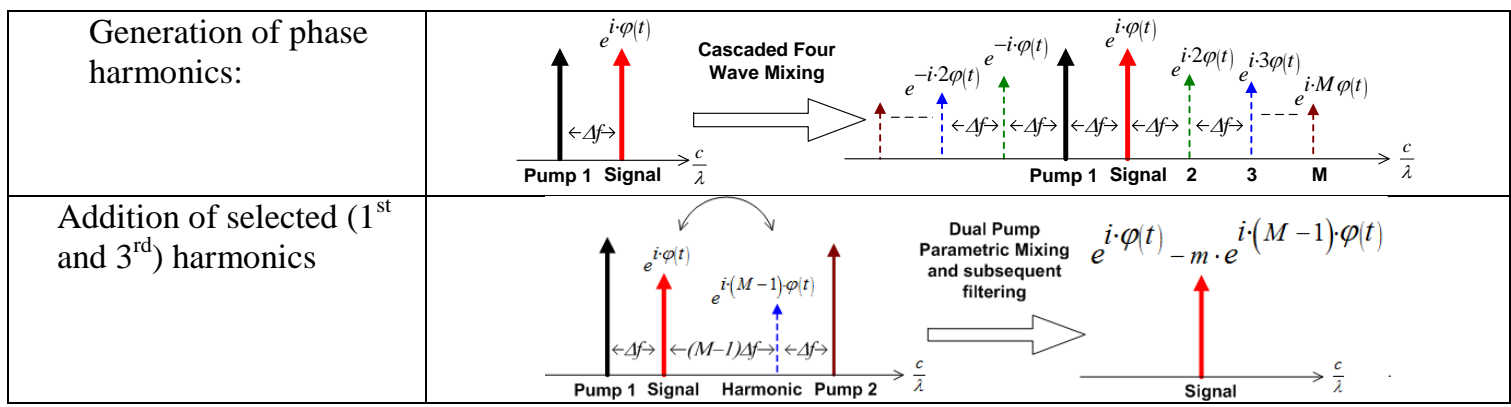

Fig. 2. Regeneration in two steps: (1) Nonlinear generation of phase harmonics via four wave mixing (FWM) [Synthesis of Fourier Components]; (2) Parametric 2-pumps non-degenerate phase sensitive amplification [Addition of selected Fourier terms]. For QPSK: M=4, for 8PSK: M=8, $\mathrm{m}$ is the scaling factor found numerically [5].

\section{Conclusions}

We discuss/show methods for direct manipulation of the optical field through a set of FWM processes. We review application for all-optical regeneration of QPSK signals. However, to be practical, it would need further research in order to allow for regeneration of wavelength multiplexed signals (WDM) and possibly also more complex modulation formats (e.g., 16 QAM), which has not been demonstrated so far and remains a topic of active research.

This research has received funding from the EU FP7 Programme under grant agreement 224547 (PHASORS) and the EPSRC under grant EP/I01196X: Transforming the Future Internet: The Photonics Hyperhighway. R. Slavík acknowledges UK Engineering and Physical Sciences Research Council, Fellowship grant agreement no. EP/K003038/1.

\section{References}

[1] J. P. Gordon, and L. F. Mollenauer, "Phase noise in photonic communications systems using linear amplifiers," Opt. Lett. 15(23), 1351-1353 (1990).

[2] K. Croussore, et al., "Demonstration of phase-regeneration of DPSK signals based on phase-sensitive amplification," Opt. Express, 13, 39453950 (2005).

[3] R. Slavík et al, "All-optical phase and amplitude regenerator for next-generation telecommunications systems," Nature Photonics 4 , 690695 (2010).

[4] J. Kakande et al, "First demonstration of all-optical QPSK signal regeneration in a novel multi-format phase sensitive amplifier," ECOC 2010 Turin 19-23, Italy, PD 3.3, Sep 2010.

[5] J. Kakande et al, "Multilevel quantization of optical phase in a novel coherent parametric mixer architecture", Nature Photonics 5, 748-752, 2011.

[6] S. Sygletos et al, “A practical phase sensitive amplification scheme for two channel phase regenerartion, Opt. Express 19, B938-B945 (2011). 